\title{
Future challenges
}

\author{
Charles Perrings*
}

School of Life Sciences, Arizona State University, P.O. Box 874501, Tempe, AZ 85287-4501

$\mathrm{T}$ he central question addressed by the papers in this special feature lies at the core of the emerging science of sustainability. What is the decision-science needed to address sustainability issues, and how does it differ from existing decision-science? The answer is not at all obvious. Many, if not the majority, of decision scientists still take the view that sustainability changes nothing but the label placed on long-standing research questions. After all, the field of resource economics has focused on the sustainable use of renewable resources since the mid-1950s (1) and on sustainable development based on the extraction of nonrenewable resources since the mid1970s (2). The basic insights that support both foci were developed even earlier (3), while concern over the sustainability of mineral extraction has been a recurrent theme since Jevons's 19th-century speculations on the coal question (4). Others argue, however, that we are seeing the beginning of a major change in the way the science of human use and impacts on the environment is being done. The focus on panaceas provides Ostrom and colleagues with a hook to explore these arguments.

Of course no one is going to argue the case for panaceas in the original sense of the word. No matter how good Panacea's own medical genes, ${ }^{\dagger}$ the application of a single cure for all diseases has long since been abandoned. The real issue is not so much that there are literal one-size-fits-all solutions being applied to the science of the environment as that the way sciences are structured encourages solutions that are insufficiently sensitive to the properties of the system or the problem being analyzed (5). There are three related points at issue, which I discuss below. The first concerns the link between sustainability science and existing disciplines. Although the development of disciplinebased science has been the source of almost all scientific advances in the last century, it has also limited the capacity of science to address problems that span multiple disciplines. The second concerns the problems posed by the increasingly tight linkages between components of the general system, often referred to as globalization. This has important implications for the behavior of the system and for the spatial and temporal scales at which environmental problems need to be addressed. Many traditional solutions address the wrong scale. The third point at issue is the growth of uncertainty in a rapidly evolving system, which also has implications for the way we think about uncertainty and learning in the decision process.

\section{Disciplines in Sustainability Science}

If there is a consensus about sustainability science, it is that the science is problem-driven and involves understanding the dynamics of evolving, coupled social-ecological systems (SESs) (6). It is inconsistent with approaches that, like panaceas, are insufficiently specific. One example that concerns Ostrom (5) is the economists' focus on market solutions to the unsustainable use of common-pool natural resources. There is indeed a natural disciplinary bias in the economists' approach to these issues, and it can look like a hammer in search of a nail. Yet there are many cases where the unsustainable use of environmental resources is driven by the lack of well functioning markets. Where the approach is less helpful, as the case studies of water, forests, and fisheries in this special feature make clear, is where there are multiple sources of failure, where understanding of the system structure and dynamics is such that rightholders will have insufficient information to manage the resource sustainably, or where local processes are driven by remote conditions (7-10). Other examples of disciplinary myopia are not hard to find. Solutions that are systematically biased in favor of technological fixes generally derive from engineers. Solutions that emphasize governance and process typically derive from political scientists. Solutions that play up the importance of culture and traditional knowledge stem from social anthropologists, and so on.

At the same time, progress has been made in the development of models and methods to address sustainability problems in coupled SESs. Early responses to the Brundtland report (11) included the formation of the International Society for Ecological Economics in 1989 to promote the development of a science that integrates the concepts, methods, and approaches of economics and ecology to be able to confront sustainability problems. Other initiatives, including the Royal Swedish Academy of Science's Beijer Institute and the Resilience Alliance, followed. Although both economics and ecology are still full of examples of disciplinary myopia, the integration of the disciplines through such ventures has generated more flexible and adaptive solutions to both the management and the science of common-pool environmental resources $(12,13)$. By focusing on the properties of coupled systems that enhance their capacity to function over a range of environmental conditions, they address a central problem of sustainability: persistence under uncertainty (14).

\section{Globalization}

The trends that most frequently test particular solutions to sustainability problems are the closer integration of the global SES, the complexity of that system, and the growing uncertainty that stems from the evolutionary nature of change. The closer integration of the world system has both social and biophysical dimensions to it. The social dimension is exemplified by the growth of world trade. The closer integration of the biophysical system is exemplified by the growth in the dispersion rate of species and the increasing homogenization of land-use patterns worldwide. Of course, the two things are linked.

Trade and travel are the mechanisms by which species are redistributed internationally (15).

Problematic solutions in this case derive from models that ignore important dimensions of the problem. The analysis of the spread of foot-and-mouth disease in the United Kingdom in 2001, for example, was based on epidemiological models that ignored the structure of livestock markets or the incentives offered by the control mechanisms and compensation schemes (16). More recently, research on the risks of an avian flu pandemic has sought to do better. Kilpatrick et al. (17) have shown that whereas the spread of the virus $\mathrm{H} 5 \mathrm{~N} 1$ in Europe is dominated by movement of wild birds, spread in Asia and Africa involves both wild bird movements and the poultry trade, and the risk to the Americas is almost entirely attributable to the poultry trade. Yet solutions proposed for most problems involving the interaction of natural and social processes are stubbornly monodisciplinary.

Many solutions also are confounded by the scale at which the problem is evalu-

\footnotetext{
Author contributions: C.P. wrote the paper. The author declares no conflict of interest. Abbreviation: SES, social-ecological system. *E-mail: charles.perrings@asu.edu.
}

†Panacea herself was goddess of healing and cures. Her father was Asclepius, god of healing and medicine. Her grandfather was Apollo, god of healing. Her two sisters were laso, goddess of recuperation, and Hygeia, goddess of disease prevention.

( 2007 by The National Academy of Sciences of the USA 
ated, both temporally and spatially, addressing short-term localized symptoms rather than the long-term dispersed drivers of change. There is, however, considerable scope for developing a science of sustainability that successfully integrates both. To take the same example of species redistribution, there is scope for connecting the insights from ecology on the significance of changes in species dispersal with insights from economics into changes in the pattern and level of world trade. In a spatially heterogeneous environment comprising a metacommunity connected through species dispersal, trade regulates the rate of dispersal and through that both the performance of each community and the degree to which they behave independently. The integration of communities that results from very high rates of dispersal leads to their homogenization, threatening the resilience or robustness, and hence the sustainability, of both the individual communities and the metacommunity $(18,19)$.

\section{Uncertainty and Learning}

The concept of bounded rationality has a long pedigree in economics (20), reflecting the empirical observation that where the costs of acquiring complete information about complex problems is high, decision-makers commonly use simple heuristics or act intuitively (21). Applying a rule of thumb makes sense when decisions are routine and the benefits of computing the optimum are small. It makes less sense when decisions involve high and potentially irreversible costs. In the present state of understanding of the dynamics of coupled SESs, there is fundamental uncertainty about both the structure of the system and the measures of its performance. ${ }^{\ddagger}$ Many coupled systems are only partially observable and controllable, and the most that may be achieved is their stabilization: the regulation of stresses on the uncontrolled part of the system to

FFundamental uncertainty implies that either the set of outcomes or the probability distribution attaching to those outcomes is not known.

1. Gordon HS (1954) J Polit Econ 62:124-142.

2. Solow RM (1974) Rev Econ Stud 41:29-46.

3. Hotelling H (1931) J Polit Econ 39:137-175.

. Jevons WS (1865) The Coal Question (Macmillan, London).

5. Ostrom E (2007) Proc Natl Acad Sci USA 104:1518115187.

6. Clark WC, Dickson NM (2003) Proc Natl Acad Sci USA 100:8059-8061

7. Berkes F (2007) Proc Natl Acad Sci USA 104:15188-15193.

8. Meinzen-Dick R (2007) Proc Natl Acad Sci USA 104:1520015205.

9. Nagendra H (2007) Proc Natl Acad Sci USA 104:1521815223.

10. Wilson J, Yan L, Wilson C (2007) Proc Natl Acad Sci USA 104:15212-15217.

11. World Commission on Environment and Development (1987) Our Common Future (Oxford Univ Press, Oxford). maintain stability given uncertainty about that part of the system. Such stabilization is complicated because policies that may be robust to uncertainty in some parameters turn out to be highly vulnerable to uncertainty in others (22). Indeed, the main scientific challenge of sustainability is to learn the dynamics of complex coupled systems without compromising their ability to deliver the things that people value.

Bayesian model averaging provides a mechanism for selecting between models that have different structural characteristics. Weighting competing models on the basis of their fit to the data, their predictive capacity, or the loss associated with decision model error provides a potentially useful mechanism for model selection. In particular, updating on measures of output dispersion, the variation in the loss function associated with a decision rule applied to different models $(23,24)$, offers an attractive tool for tracking model performance.

One of the most telling outcomes of the recent Millennium Ecosystem Assessment (25) was the conclusion that although we understand the linkages among biodiversity, ecosystem functioning, ecosystem services, and human well being, and although we have some understanding of the linkages between human behavior and biodiversity loss, we are next to helpless to predict the consequences of alternative strategies for managing changes in biodiversity. Because the assessment argued strongly that anthropogenic factors lie behind the loss of biodiversity and consequential changes in ecosystem services, and hence that policy changes are needed to reverse the trend, the conclusion is worrisome.

The two options for dealing with the uncertain future consequences of human activities, mitigation and adaptation, have fundamentally different implications both for the distribution of the benefits of current decisions and for science. Whereas mitigation generally implies action now to protect the interests of future generations everywhere (it is a public good), adapta-

12. Wätzold F, Drechsler M, Armstrong CW, Baumgärtner S, Grimm V, Huth A, Perrings C, Possingham H, Shogren J, Skonhoft A, et al. (2006) Conserv Biol 20:1034-1041.

13. Walker BH, Anderies JM, Kinzig AP, Ryan P (2006) Ecol Soc 11(1):12.

14. Perrings C (2006) Environ Dev Econ 11:417-427.

15. Perrings C, Dehnen-Schmutz K, Touza J, Williamson M (2005) Trends Ecol Evol 20:212-215.

16. Royal Society (2002) Infectious Diseases in Livestock (Royal Society, London).

17. Kilpatrick AM, Chmura AA, Gibbons DW, Fleischer RC, Marra PP, Daszak P (2006) Proc Natl Acad Sci USA 103:19368-19373.

18. Loreau M, Mouquet N, Gonzalez A (2003) Proc Natl Acad Sci USA 22:12765-12770.

19. Levin SA (2000) Fragile Dominion (Perseus, Reading, MA) 20. Simon H (1957) Models of Man (Wiley, New York). tion provides benefits to those who adapt (it is a private good). Mitigation also demands at least some capacity to predict the consequences of present actions; adaptation does not. Unlike adaptation, mitigation decisions are not supported by nonprobabilistic scenarios. Indeed, for some at least, the holy grail of sustainability science is the improvement of our capacity to predict at least components of the system as a precondition for mitigating the risks to future generations. The task gets harder, not easier, as those systems evolve. But at least there are methods for selecting among competing models of system dynamics and for systematic learning about model error.

Although there is still no way to anticipate surprises in an evolutionary system, there are methods for identifying early warnings of threshold effects that are signaled in the data. In particular, wherever threshold effects are anticipated by changes in the volatility of particular system measures, analysis of the spectral properties of the data provides a potential early warning of impending change (26). Detection still depends on the existence of appropriate models of system dynamics, i.e., on an understanding of which measures matter and why. It also still leaves the problem of communicating the results of science in ways that are both compelling to decision-makers and timely $(27,28)$.

The principal challenge in building a science of sustainability is the development of predictive models of system change that enable society at least to evaluate mitigation options alongside adaptation. Building sustainability science is about building capacity, methods, and protocols to analyze problems stemming from the dynamics of complex coupled SESs. One part of that task is figuring out how to break existing disciplinary biases about concepts, methods, and analysis. A second is to induce reappraisal of the rules of thumb that structure both research and decision making. Ostrom and colleagues have already done much to address the first, and they are on their way to addressing the second.

21. Kahneman D (2003) Am Econ Rev 93:1449-1475 22. Anderies JM, Rodriguez AA, Janssen MA, Cifdaloz O (2007) Proc Natl Acad Sci USA 104:15194-15199.

23. Brock WA, Carpenter SR (2007) Proc Natl Acad Sci USA 104:15206-15211

24. Brock WA, Durlauf SN, West KD (2007) J Econometrics, in press.

25. Millennium Ecosystem Assessment (2005) Ecosystems and Human Well-Being: Synthesis (Island, Washington, DC).

26. Brock WA, Carpenter S (2006) Ecol Lett 9:311-318.

27. Harremoës P, Gee D, MacGarvin M, Stirling A, Keys J, Wynne B, Guedes Vaz S, eds (2001) Late Lessons from Early Warnings (Environment Issue Report No. 22) (European Environment Agency, Copenhagen).

28. Loreau M, Oteng-Yeboah A, Arroyo MTK, Babin D, Bar bault R, Donoghue M, Gadgil M, Häuser C, Heip C, Larigauderie A, et al. (2006) Nature 442:245-246. 